Chlamydomonas; EDTA at a concentration of $5 \times 10^{-5} \mathrm{M}$ and $10^{-5} \mathrm{M}$ had the same effect as IAA. At higher concentrations, $10^{-4} \mathrm{M}$ and above, EDTA inhibits motility of the organism. The action of EDTA was not so sharply $p \mathrm{H}$ dependent as that of IAA, being equally effective at $p \mathrm{H} 6.0$ and 5.6 at a concentration of $5 \times 10^{-5} \mathrm{M}$.

It is at present difficult to conjecture about the possible mode of action of IAA and EDTA in indueing phototactic responses and in reversing the inhibitory action of acetic acid. Probably IAA does not act here as a conventional hormone. Although both IAA and EDTA can act as chelates, for example, of calcium it seems unlikely that they do so here, in view of the $p \mathrm{H}$ range in which they are active and the very weak chelating capacity of IAA. However, it must be noted that IAA and EDTA may be regarded as derivatives of acetic acid. This, together with the interaction of IAA with the latter, makes it possible that they compete for some specific site within the flagella or cell. This site would determine the correct translation of the impulses sent from the light receptor into the flagella. Correct translation results in flagellar beat such as to orient the organism toward the light; prevention of translation also prevents oriented movement.

Department of Botany,

Hebrew University, Jerusalem.

${ }^{1}$ Stahl, N., and Mayer, A. M., Science, 141, 1282 (1963).

${ }^{2}$ Mayer, A. M., and Poljakoff-Mayber, A., Phys. Plant, 12, 8 (1959).

${ }^{3}$ Sachs, T., and Mayer, A. M., Phycologia, 1, 150 (1961).

\section{Actions and Dosage of Chloralose}

MANY books recommend chloralose as an anæsthetic for laboratory animals and give a dosage rate of 40-80 $\mathrm{mg} / \mathrm{kg}$ body-wt.; the drug is considered particularly useful for animals in which autonomic reflexes are to be preserved. I have recently carried out a series of experiments in the dog and rabbit, and in no case did surgical anæsthesia result from intravenous injections of $70 \mathrm{mg} / \mathrm{kg}$ or less. If chloralose was used after induction with ether slight improvement was seen, but surgery was still not possible. The heart rate was slowed by 30 per cent in these experiments and excessive secretions caused respiratory embarrassment.

The action of chloralose has not been well understood in the past: "The action is a remarkable one, for the animal does not appear to pass beyond a stage of narcosis, and paddling movements of the legs or nodding of the head may appear throughout. . . . It is suggested that the condition is possibly one of hypnosis superimposed on narcosis, rather than anæsthesia". Electrocorticographic recordings now show that it makes the Betz cells susceptible to sensory stimuli2, and that the convulsive response to slight stimulus which readily occurs under chloralose is a cortical reaction, for "chloralose, like the convulsants, facilitates transmission in a reflex are including the cortex"3. Grieg ${ }^{4}$ described these convulsions as "strychnine-like". Shukla et al.s, studying its effect on autonomic roflexes, found that it slowed the heart rate by 50 per cent, while Kochmann 6 found that "it roinforces some actions of epinephrine and acts like atropine in reducing vagal activity".

In viow of the fact that chloralose does not affect the electrical activity of the brain in the same way as other drugs known to be general anæsthetics, and bearing in mind that it does not leave autonomic reflexes unaltered, it would seem that some substitute should be sought for this drug; a great variety of new substances acting on the central nervous system have been developed since the Socond World War, and traditional procedures may not be justified in the light of present knowledge. The fact that chloralose is not used in human medicine casts further doubt on the validity of its use as an anæsthetic for laboratory animals.

Universities Federation for Animal Wolfare,

$7 a$ Lamb's Conduit Passage, London, W.C.1.

${ }^{1}$ Wright, J. G., and Hall, L. W., Veterinary Ancesthesia, flfth ed., 209 (Bailliere, Tindall and Cox, London, 1961)

'Adrian, E. D., and Moruzzi, G., J. Physiol., 97, 153 (1939).

${ }^{3}$ Hiil, D., and Parr, G., Electroencephalography, first ed., 184 (MacDonald, London, 1950).

- Grieg, J. R., Hoare's Veterinary Medicine and Therapeutics, fifth ed., 194 (Bailiiere, Tindall and Cox, London, 1933).

- Shukla, R. C., et al., Indian J. Physiol., 16, 17 (1962).

- Kochmann, review quoted in Sollmann's Manual of Pharmacology (Saunders, London, 1936).

\section{Some Negative Results in the Search for a Lethal Effect of Magnetic Fields on Biological Materials}

DURING recent years there has been an increasing number of reports in the literature of the ability of magnetic fields to affect biological systems. Gerencser and Barnothy ${ }^{1}$ noted differences from control of cultures exposed to 15,000 gauss, while Butler and Dean ${ }^{2}$ found growth inhibition in tissue culture of $K B$ cells exposed to 4,000-gauss magnetic fields. Maclean ${ }^{3}$, using intense and 'mild' magnetic fields of unspecified strengths, reported various effects on tumour-bearing mice, but since only 12 animals were used for the entire investigation and these were divided into 3 groups, the results can have no statistical significance. Negative reports have appearod too, and in the most recent of these Halpern and Green $\theta^{4}$ reported that the growth rate of HeLa cells cultured in a 1,200 -gauss magnetic field was not significantly different from controls. This communication extends this negative finding to much higher magnetic field strengths. Three separate experiments were performed, using HeLa cells cultured in vitro.

The cells were cultured by the methods described elsewhere ${ }^{5}$. In each experiment 200 cells were inoculated into either $50 \mathrm{~mm}$ 'Falcon' plastic Petri dishes or into 'Falcon' plastic culture flasks. Two containers were reserved for controls and two were subjected to a magnetic field for various lengths of time as detailed here. The cells were afterwards incubated for 10 days at $37^{\circ} \mathrm{C}$ in an incubator continuously flushed with a mixture of 5 per cent carbon dioxide and 95 per cent air. At the end of this period the cells were fixed with 10 per cent formalin in normal saline, stained with crystal violet, and the number of macroscopic colonies on each dish or flask counted. The following treatment conditions were used.

(a) A steady field of 5,000-gauss was obtained with a permanent magnet having 2 -in. diameter pole pieces which were just large enough to cover the $50 \mathrm{~mm}$ plastic Petri dishes. The magnet was mounted inside the incubator and the treated platos were subjected to the magnetic field throughout the 10-day period of incubation. The control plates were kept in the same incubator at approximately 12 in. from the pole pieces. Approximately 150 colonies were counted in each dish, but thero was no significant difference between the number of colonies counted on the treated against the control dishes (Table 1). There was no obvious difference in the size of the colonies, or their morphology.

$(b, c)$ To obtain stronger magnetic fields, it was necessary to use electromagnets. Consequently it was not possible to mount the magnets inside the incubator and, for this and other reasons, treatment times were much shorter.

In one exporiment an electromagnet was used which had tapered pole pieces, 4-in.--2-in. diameter, with a gap between the poles of $15 \mathrm{~mm}$. Two culture flasks were exposed to a magnetic field of 27 kilogauss for a period of 